\title{
Passive immunity to porcine epidemic diarrhea virus following immunization of pregnant gilts with a recombinant orf virus vector expressing the spike protein
}

\author{
Lok R. Joshi ${ }^{1,2} \cdot$ Faten A. Okda ${ }^{1}$. Aaron Singrey ${ }^{1,2} \cdot$ Mayara F. Maggioli $^{1,2} \cdot$ Tatiane C. Faccin $^{3,1}$. \\ Maureen H. V. Fernandes ${ }^{1,2} \cdot$ Kyle S. Hain ${ }^{1}$ - Scott Dee ${ }^{4}$. Fernando V. Bauermann ${ }^{1,2}$. Eric A. Nelson ${ }^{1,2}$. \\ Diego G. Diel ${ }^{1,2}$ (D)
}

Received: 4 January 2018 / Accepted: 18 April 2018 / Published online: 3 May 2018

(c) Springer-Verlag GmbH Austria, part of Springer Nature 2018

\begin{abstract}
Passive immunity is critical for protection of neonatal piglets against porcine epidemic diarrhea virus (PEDV). Here, we investigated the immunogenicity of an orf virus (ORFV) vector expressing the full-length spike (S) protein of PEDV (ORFVPEDV-S) in pregnant gilts and its ability to confer passive immunity and protection in piglets. Three doses of ORFV-PEDV-S were given to two groups of PEDV-negative pregnant gilts, with the last dose being administered two weeks prior to farrowing. One of the two groups immunized with the ORFV-PEDV-S recombinant virus was also exposed to live PEDV orally on day 31 post-immunization (pi). Antibody responses were assessed in serum, colostrum and milk of immunized gilts, and passive transfer of antibodies was evaluated in piglet sera. The protective efficacy of ORFV-PEDV-S was evaluated after challenge of the piglets with PEDV. PEDV-specific IgG, IgA and neutralizing antibody (NA) responses were detected in ORFV-PEDV-S-immunized and ORFV-PEDV-S-immunized/PEDV-exposed gilts. PEDV NA, IgG and IgA were detected in the serum of piglets born to immunized gilts, demonstrating the transfer of antibodies through colostrum and milk. Piglets born to immunized gilts showed reduced morbidity and a marked reduction in mortality after PEDV challenge in comparison to control piglets. Piglets born to gilts that received ORFV-PEDV-S and were exposed to live PEDV showed stronger NA responses and lower clinical scores when compared to piglets born to gilts immunized with ORFV-PEDV-S alone. These results demonstrate the potential of ORFV as a vaccine delivery platform capable of eliciting passive immunity against PEDV.
\end{abstract}

Handling Editor: YiMing Shao.

Diego G. Diel

diego.diel@sdstate.edu

1 Department of Veterinary and Biomedical Sciences, Animal Disease Research and Diagnostic Laboratory, 1155 North Campus Drive, Box 2175, Brookings, SD 57007, USA

2 Center for Biologics Research and Commercialization, South Dakota State University, Brookings, SD 57007, USA

3 Programa de Pós-Graduação em Medicina Veterinária, Universidade Federal de Santa Maria, Santa Maria, RS 97105-900, Brazil

4 Pipestone Veterinary Services, Pipestone Applied Research, Pipestone, MN 51164, USA

\section{Introduction}

Porcine epidemic diarrhea virus (PEDV), an alphacoronavirus in the family Coronaviridae, is a single-stranded, positive-sense RNA virus that causes porcine epidemic diarrhea (PED) in pigs [1]. PEDV infects pigs of all age groups resulting in an enteric disease with high morbidity. High mortality rates (50\%-100\%) are observed in neonatal piglets; however, mortality is usually low in older animals [2]. PED is characterized by vomiting, watery diarrhea and dehydration, which are usually followed by death in suckling piglets [3]. After its introduction in the USA in 2013, PEDV caused the deaths of over 7 million piglets, resulting in significant economic losses to the US swine industry [4]. Since then, significant investments and efforts to develop PEDV vaccines have been made, with a few vaccines receiving a conditional license from the USDA. The efficacy of these vaccines in protecting newborn piglets, however, is still unknown. 
Piglets are born agammaglobulinemic due to the impermeable nature of the epitheliocorial swine placenta, and their immune system is immature, which makes them highly susceptible to PEDV in the first weeks (1-3) of life. Therefore, passive transfer of antibodies through colostrum and milk is critical for protection of neonatal piglets against PEDV $[5,6]$. Previous studies with transmissible gastroenteritits virus (TGEV), a coronavirus that is closely related to PEDV, have shown a high correlation between milk antibody levels and protection in piglets [7, 8]. Hence, a requirement for an effective PEDV vaccine is the ability to induce high levels of antibodies in colostrum and milk with their subsequent transfer to piglets born to immunized sows. Although several attenuated, killed, or subunit PEDV vaccines have been developed, most of them fail to induce sufficient levels of lactogenic immunity and protection in newborn piglets [9, 10]. Thus, it is critical to develop improved alternatives to currently available PEDV vaccines.

Recently, we demonstrated that a recombinant orf virus (ORFV) expressing the full-length spike (S) protein of PEDV (ORFV-PEDV-S) is capable of eliciting protective immune responses in immunized pigs [11]. Three-week-old piglets immunized intramuscularly (IM) with the ORFVPEDV-S recombinant virus were protected from clinical signs of PED after oral challenge with PEDV and showed reduced virus shedding in feces [11]. In the present study, we investigated the immunogenicity of ORFV-PEDV-S recombinant virus in pregnant gilts and its ability to induce passive immunity and protection in piglets born to immunized animals.

\section{Materials and methods}

\section{Viruses and cells}

The recombinant ORFV-PEDV-S was previously generated and characterized in our laboratory [11], propagated and titrated in primary ovine fetal turbinate cells (OFTu, provided by D.L. Rock, University of Illinois) [11]. PEDV strain USA/CO/2013 (CO13) was obtained from the National Veterinary Services Laboratory (NVSL) and propagated in Vero-76 cells (ATCC ${ }^{\circledR}$ CRL-1587 ${ }^{\mathrm{TM}}$ ) in the presence of
$1.5 \mu \mathrm{g}$ of TPCK-treated trypsin per mL (Sigma Aldrich, St. Louis, MO).

\section{Immunization of pregnant gilts and challenge studies}

Six primiparous gilts were randomly assigned to three experimental groups as follows: group $1(\mathrm{G} 1)$, control (MEM; $\mathrm{n}=2$ ); group $2(\mathrm{G} 2)$, ORFV-PEDV-S-immunized $(\mathrm{n}=2)$; group $3(\mathrm{G} 3)$, ORFV-PEDV-S-immunized/live PEDV-exposed $(\mathrm{n}=2)$ (Table 1). Animals from $\mathrm{G} 2$ and G3 were immunized intramuscularly (IM) with $2 \mathrm{ml}$ of the recombinant ORFV-PEDV-S containing $10^{7.38}$ tissue culture infectious dose $50\left(\mathrm{TCID}_{50}\right) / \mathrm{mL}$ in MEM. All animals received the first immunization on day 0 and two booster immunizations on days 21 and 42 post-immunization (pi). The second booster was administered two weeks prior to the anticipated parturition date. In addition to the immunization regimen with ORFV-PEDV-S described above, gilts in G3 were exposed to live PEDV orally $\left(1 \times 10^{5} \mathrm{TCID}_{50}\right.$ in 1 $\mathrm{ml}$ ) on day 31 pi. Animals from G1 were sham-immunized with $2 \mathrm{ml}$ MEM as described above. Animals were housed in BSL-2 animal rooms, and two weeks prior to farrowing, each gilt was transferred to individual farrowing crates (two crates per room).

All gilts received prostaglandin- $\mathrm{F}_{2 \alpha}(10 \mathrm{mg})$ on day $52 \mathrm{pi}$ by IM injection to induce parturition. Animals from G1 and G3 farrowed on day $53 \mathrm{pi}$, while animals from $\mathrm{G} 2$ farrowed on day 56 pi. Twelve piglets from each sow were randomly selected, kept with their mothers $(n=24$ per group) for 17 days and allowed to suckle colostrum and milk ad libitum. Excess piglets were euthanized to keep all sows with an equal number of piglets $(n=12)$. All piglets $(\mathrm{G} 1, \mathrm{G} 2$ and G3) were challenged orally on day 7 post-birth with a virus suspension containing $2.5 \times 10^{2} \mathrm{TCID}_{50}$ of PEDV strain $\mathrm{CO} 13$ (1 ml/piglet). Animals were monitored daily for clinical signs and mortality, and the experiment was terminated on day 74 pi or day 11 post-challenge (pc). Clinical scores were evaluated based in four criteria, which were modified from a scoring method described by Lohse and collaborators [12] as follows: a) well-being: normal $=0$, slightly depressed $=1$, depressed and lethargic $=2 ; b$ ) defecation: normal feces $=0$, semi-solid and pasty $=1$, watery feces $=2$; c) vomiting: no $=0$, yes $=1 ; d$ ) body condition: normal $=0$,

Table 1 Experimental design

\begin{tabular}{lllll}
\hline Group (n) & Treatment & $\begin{array}{l}\text { Day of immuniza- } \\
\text { tion (route) }\end{array}$ & Dose TCID $_{50}$ & No of piglets \\
\hline Group 1 (G1) $(\mathrm{n}=2)$ & Control (MEM) & $0,21,42(\mathrm{IM})$ & $2 \times 10^{7.38}$ & 24 \\
Group 2 (G2) $(\mathrm{n}=2)$ & ORFV-PEDV-S & $0,21,42(\mathrm{IM})$ & $2 \times 10^{7.38}$ & 24 \\
Group 3 (G3) $(\mathrm{n}=2)$ & ORFV-PEDV-S & $0,21,42(\mathrm{IM})$ & $2 \times 10^{7.38}$ & 24 \\
& Live PEDV & 31 (oral) & $1 \times 10^{5}$ & \\
\hline
\end{tabular}


thin $=1$, emaciated $=2$. Mean daily group scores and mortality rates were calculated and compared between different groups. Piglets showing severe dehydration and emaciation were euthanized based on the independent evaluation of SDSU's veterinarian.

Serum was collected from sows on days $0,21,28,35$, 42, 49, 54, 60, 63, 67, 70 and 74 pi. Additionally, colostrum and milk were collected from sows on days 1 (day of farrowing) $3,7,10,14,17$ post-farrowing. Serum and rectal swabs were collected from piglets on days 1 (pre-colostrum), 7 (pre-challenge), 10, 14, and 17 post-birth. All animal studies were conducted at the SDSU Animal Resource Wing (ARW), following the guidelines and protocols approved by the SDSU Institutional Animal Care and Use Committee (IACUC approval no. 16-003A).

\section{Antibody isotype ELISA}

Indirect ELISAs using a truncated S protein (aa 630-800) were used to assess IgG and $\operatorname{IgA}$ antibody responses in animals immunized with the recombinant ORFV-PEDV-S virus as described previously [11].

\section{Fluorescent focus neutralization assay}

Neutralizing antibody responses elicited by immunization with the recombinant ORFV-PEDV-S were assessed by fluorescent focus neutralization assay (FFN) as described previously [13].

\section{Fluorescent microsphere immunoassay (FMIA)}

PEDV S-specific IgG and $\operatorname{IgA}$ antibody responses were assessed in colostrum and milk by FMIA. Optimal assay conditions (amount of antigen, colostrum/milk and secondary antibody dilutions) were determined by a checkerboard titration [13].

\section{Real-time reverse transcription PCR (RT-qPCR)}

Virus shedding was assessed in rectal swabs by RT-qPCR. Viral RNA was extracted from rectal swabs using a Zymo Viral RNA Extraction Kit (Zymo Research, CA, USA) according to the manufacturer's instructions. Primers and a probe targeting the PEDV nucleocapsid protein were designed using PrimerQuest Tool (Integrated DNA Technologies Inc., USA). RT-qPCR was performed using a SensiFast ${ }^{\mathrm{TM}}$ Probe Lo-ROX One-Step Kit (Bioline, MA, USA) following the manufacturer's instructions. Genome copy numbers per $\mathrm{ml}$ were determined using a relative standard curve method. The amount of viral RNA detected in feces was expressed as $\log _{10}$ genome copies $/ \mathrm{ml}$.

\section{Results}

\section{Systemic antibody responses in gilts}

Gilts from both immunized groups (G2 and G3) developed PEDV S-specific IgG and IgA responses (Fig. 1A and $\mathrm{B})$. IgG and $\operatorname{IgA}$ antibodies were first detected on day 21 pi in animals in G2, and the highest level of antibodies was detected on day 35 pi. Similarly, in G3, IgG and IgA antibodies were first detected on day $21 \mathrm{pi}$, and their levels increased after the booster immunization on day 21. An anamnestic antibody response was observed in G3 gilts after they were exposed to live PEDV on day 31 pi (Fig. 1A and B). No spike-specific IgG or IgA antibodies were detected in the serum of control sows (G1).

The ability of ORFV-PEDV-S to induce neutralizing antibody (NA) responses against PEDV was assessed using a FFN assay. NA were first detected in serum a week after the first booster immunization (day 28 pi; Fig. 1C). An increase in NA levels was observed in both immunized groups (G2 and G3) after day 28 pi (Fig. 1C). Gilts in G2, which were immunized only with ORFV-PEDV-S, had the highest neutralizing antibody titers on day 49 pi, a week after the second booster immunization (day 42 pi; Fig. 1C), whereas gilts in G3, which were immunized with ORFV-PEDV-S and exposed to live PEDV (day $31 \mathrm{pi}$ ), had the highest antibody titers on day 35 pi, with titers remaining constant thereafter until the end of the experiment (Fig. 1C). Similar to the IgG and IgA responses, higher neutralizing antibody responses were observed in gilts in G3 when compared to G2 animals. No neutralizing antibodies against PEDV were detected in control gilts (G1) in serum samples collected pre-farrowing/pre-challenge. Animals in G1 seroconverted to PEDV, presenting detectable levels of IgG, IgA and NA a week after challenge of the piglets (day 7 post-birth; Fig. 1). Notably, a strong correlation between $\mathrm{NA}$ and $\operatorname{IgG}$ and $\operatorname{IgA}$ levels was observed in sow serum (NA vs. IgG: $r=0.876, P<0.0001$; NA vs. $\operatorname{IgA}: r=0.822, P<0.0001$; Fig. $1 \mathrm{D}$ and E). These results confirmed our previous findings demonstrating the immunogenicity of ORFV-PEDV-S in pigs [11].

\section{Detection of S-specific IgG and IgA antibodies in colostrum and milk}

High levels of S-specific IgG antibodies were detected on days 1 and 3 post-farrowing, decreasing thereafter in animals from G2 and G3 (Fig. 2A). Colostrum collected from sows in G3, exposed to live PEDV, had higher levels of $\mathrm{IgG}$ when compared to $\mathrm{G} 2$ on day 1 post-farrowing (Fig. 2A). High levels of S-specific IgA antibodies were 


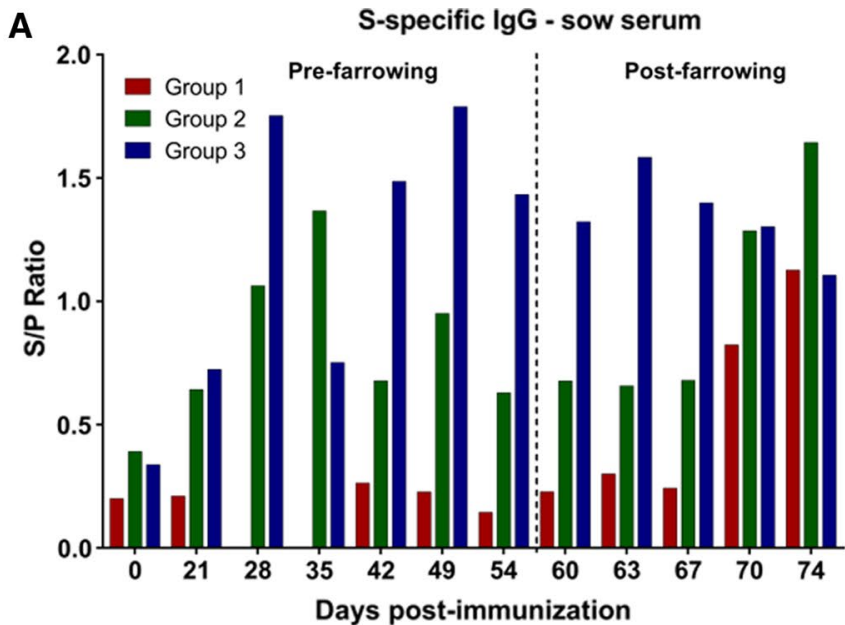

C
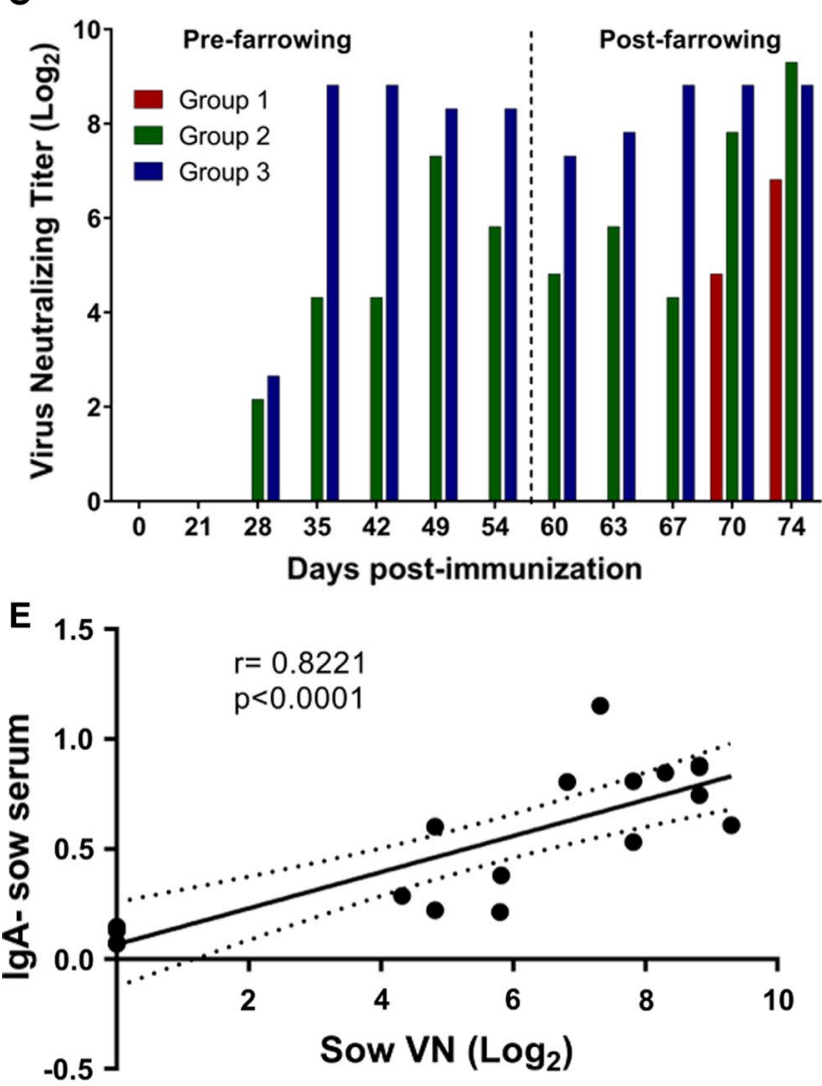

Fig. 1 Spike (S)-specific antibody responses in sows. (A) Isotype ELISA demonstrating serum IgG antibody responses specific for the PEDV S protein. (B) Isotype ELISA demonstrating serum IgA antibody responses specific for the PEDV S protein. (C) Virus neutralizing antibody (NA) responses in sows. S/P, sample-to-positive ratio. Group 2 and group 3 gilts were immunized on days 0, 21 and 42 with ORFV-PEDV-S. Oral exposure to live PEDV in group 3 gilts was

also detected in colostrum of animals from G2 and G3 on day 1 post-farrowing (Fig. 2B). Notably, the levels of $\mathrm{S}$-specific IgA antibodies remained elevated in milk up
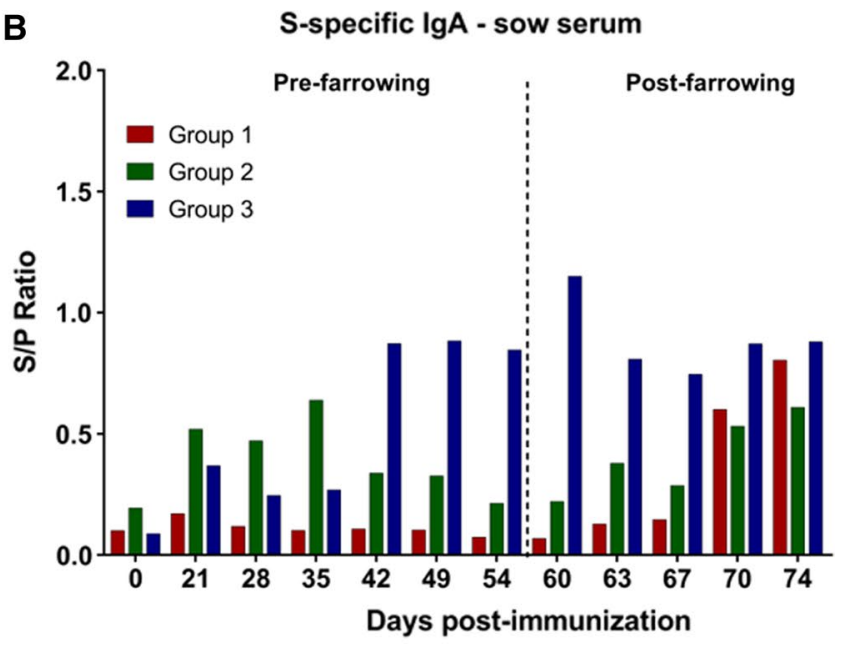

D

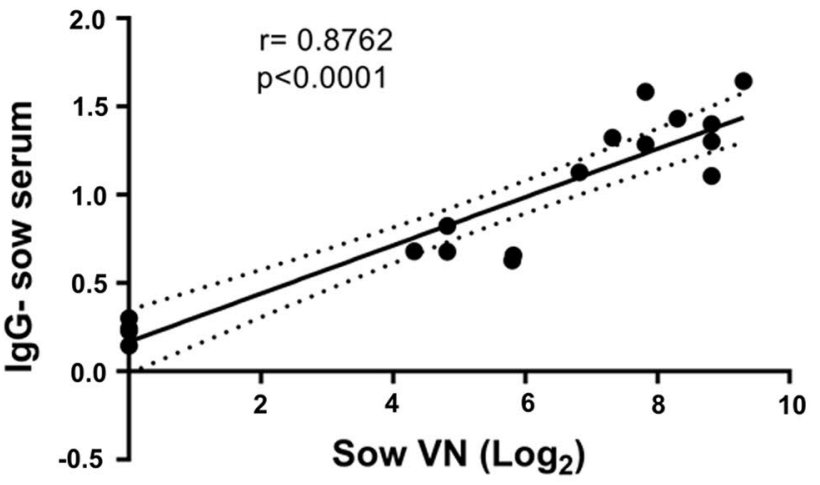

performed on day 31 pi. Group 1 control gilts were sham-immunized with minimal essential medium (MEM) on the same days. (D) Correlation of sow serum IgG levels with sow mean NA levels. (E) Correlation of sow serum IgA levels with piglet serum NA levels. Correlations were calculated using the Spearman method with a $95 \%$ confidence interval using GraphPad Prism 7

to day 17 post-farrowing (Fig. 2B). IgA antibody levels were higher in milk from animals in G3 when compared to animals in G2 after day 7 post-farrowing (Fig. 2B). 


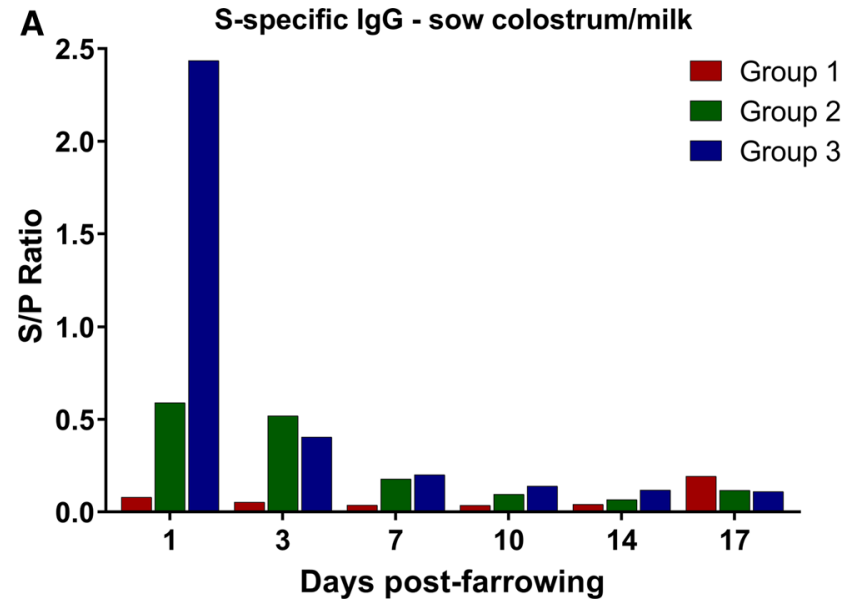

Fig. 2 Detection of PEDV S-specific antibodies in colostrum/milk. (A) Isotype fluorescent microsphere immunoassay (FMIA) demonstrating S-specific IgG antibodies in colostrum/milk of immunized

No spike-specific $\operatorname{IgG}$ or IgA antibodies were detected in colostrum/milk collected from control sows (G1) (Fig. 2A and $\mathrm{B})$. These results demonstrate the ability of ORFVPEDV-S to induce $\mathrm{S}$-specific $\operatorname{IgG}$ and $\operatorname{Ig} \mathrm{A}$ antibodies in colostrum and milk.

\section{Passive transfer of PEDV-specific antibodies to piglets}

Passive transfer of antibodies from immunized sows to their offspring was assessed by ELISA and FFN assays performed on serum samples collected from piglets pre- and post-ingestion of colostrum/milk. No S-specific antibodies were detected in serum samples collected on day one prior to ingestion of colostrum (Fig. 3). Notably, high levels of $\mathrm{S}$-specific IgG and IgA antibodies were detected in the serum of piglets from $\mathrm{G} 2$ and $\mathrm{G} 3$ on day 3 post-birth. Piglets born to immunized sows had IgG and $\operatorname{IgA}$ antibodies in their serum until the end of the experiment on day 17 post-birth (Fig. 3), with higher levels of IgG antibodies being detected until the end of the experiment. Both IgG and IgA levels were higher in piglets born to sows in G3 when compared to piglets born to sows in G2. No spike-specific IgG or IgA was detected in serum of piglets born to control sows (G1; Fig. 3).

In addition to $\operatorname{IgG}$ and $\operatorname{IgA}$, NAs were detected in piglet serum on day 3 post-birth. High levels of NA were detected in piglets born to ORFV-PEDV-S-immunized sows until the end of the experiment on day 17 pi (Fig. 3C). No neutralizing antibodies were detected in the piglets born to control sows (G1, Fig. 3C). Notably, a significant correlation between NA titers in sow serum and piglet serum was observed ( $r=0.5157, P=0.0285$ ). Additionally, a strong correlation between NA and IgG and IgA antibody

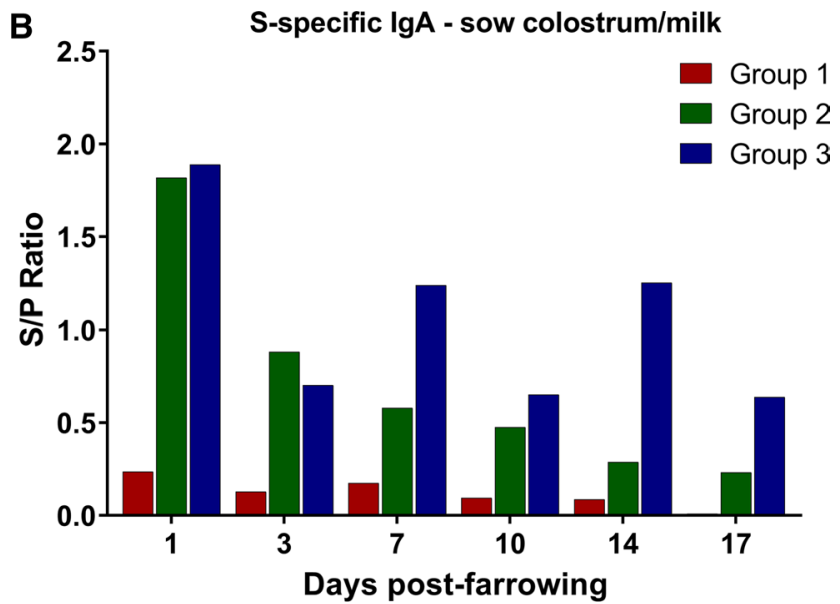

or control gilts. (B) Isotype FMIA demonstrating S-specific IgA antibodies in colostrum/milk of immunized or control gilts. S/P, sampleto-positive ratio

levels was observed in piglet serum (NA vs. IgG: $r=0.866$, $P<0.0001 ; r=0.641$; Fig. $3 \mathrm{E}$ and F). Together, these results demonstrate passive transfer of PEDV-specific IgG, IgA and NAs from immunized sows to piglets through ingestion of colostrum and/or milk.

\section{Protection of piglets against PEDV challenge}

All piglets born to immunized or control gilts were challenged orally with virulent PEDV strain CO13 on day 7 post-birth. Piglets were monitored daily for characteristic clinical signs of PED and mortality. Daily average clinical scores were calculated for each group, and the mean daily scores are presented in Fig. 4A. All piglets born to sows in G1 and G2 showed clinical signs of PED starting on day 1 pc which lasted until day 9-10 pc. The highest clinical scores were observed in G1 piglets between days 2 and 5 pc. Piglets in G3 started showing clinical signs on day $3 \mathrm{pc}$, when moderate diarrhea was observed and continued until day 11 pc. As shown in Fig. 4A, more-severe clinical signs were observed in piglets from G1 (average daily scores ranging from 4 to 7), followed by G2 (average daily scores ranging from 4 to 6) and then G3 (average daily scores ranging from 2 to 3.5). It is important to note that, while piglets in G1 and G2 experienced vomiting and diarrhea, none of the piglets in Group 3 experienced vomiting during experiment.

Virus shedding in feces was assessed by RT-qPCR using rectal swabs. Piglets in G3 had a significantly lower level of viral RNA in their feces $(P<0.0001)$ in comparison to piglets in G1 and G2 on day 3 pc (Fig. 4B). However, no significant differences in virus shedding in feces were observed between the three groups thereafter.

The daily mortality recorded for piglets in the three groups is presented in Fig. 4C. Notably, twelve out of 24 


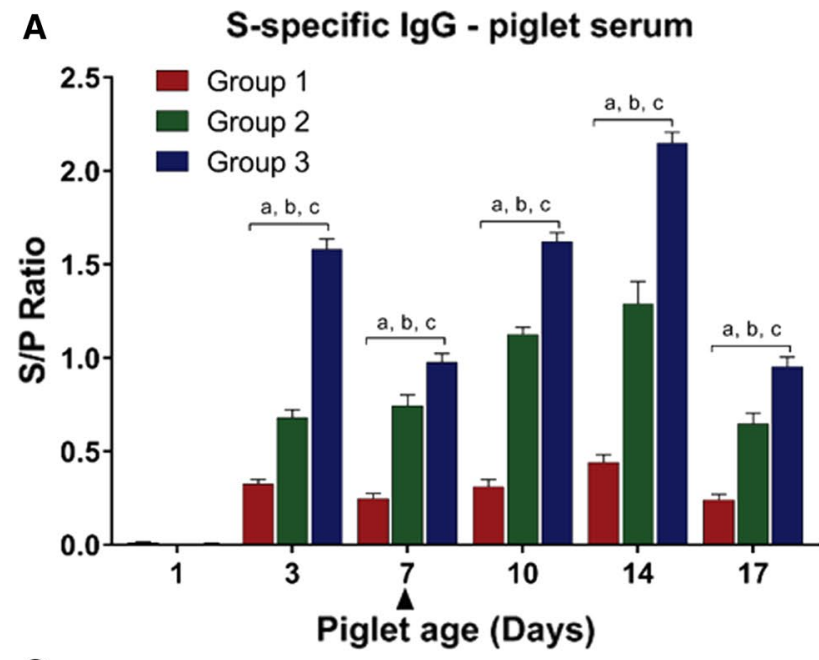

C

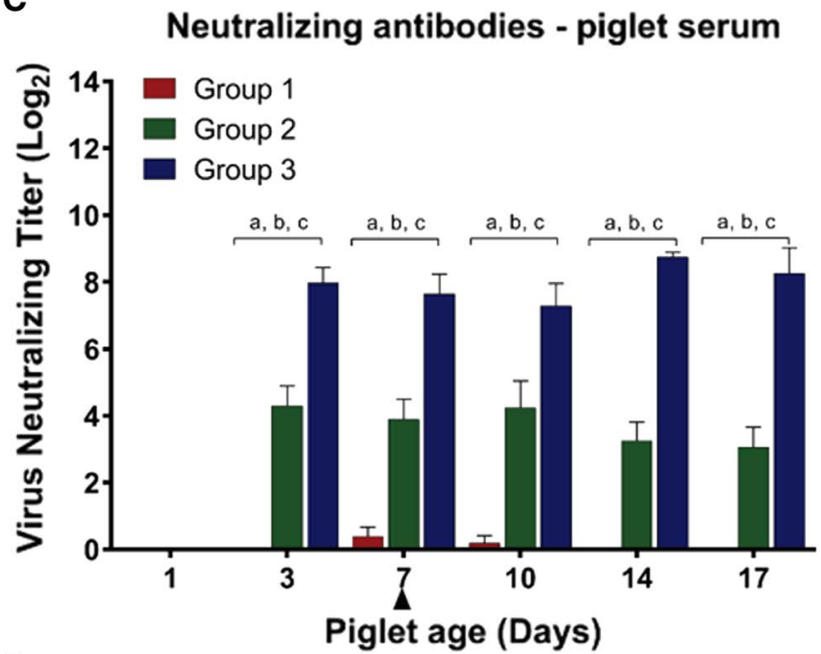

E

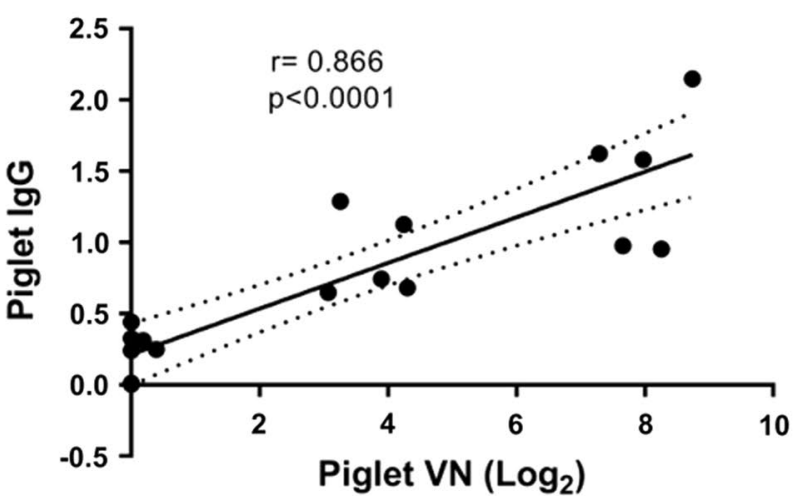

Fig. 3 Passive transfer of antibody to piglets. (A) Isotype ELISA demonstrating S-specific IgG antibody levels in piglet serum. (B) Isotype ELISA demonstrating S-specific IgA antibody levels in piglet serum. (C) Virus neutralizing antibody (NA) levels in piglet serum. Results are presented as group mean S/P ratios or NA titers. The error bars represent +/- standard error of mean (SEM). Statistical significance was determined using two-way ANOVA, and multiple comparisons were ran using Tukey's test. a, b, and c represent statistical significance for G1 vs. G2, G1 vs. G3, and G2 vs. G3, respectively. The

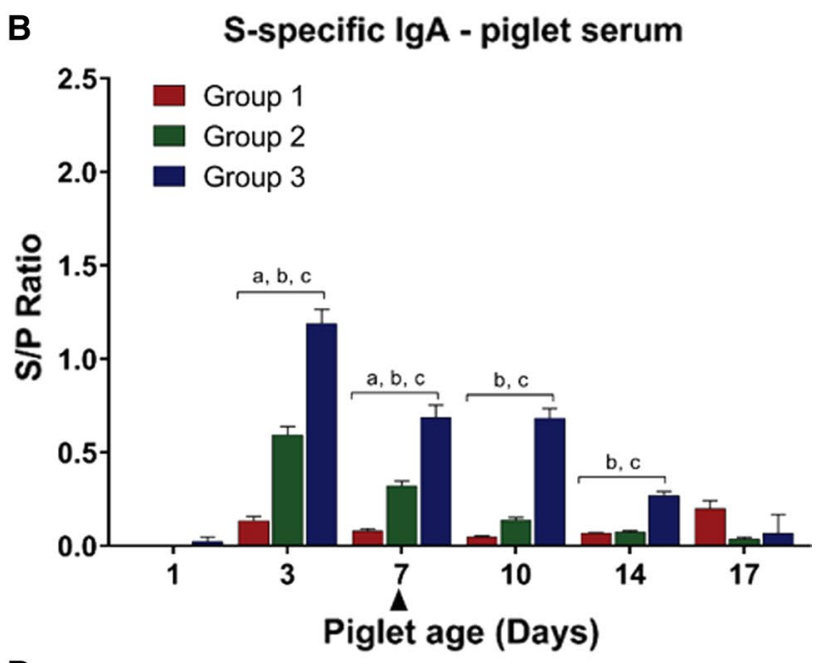

D
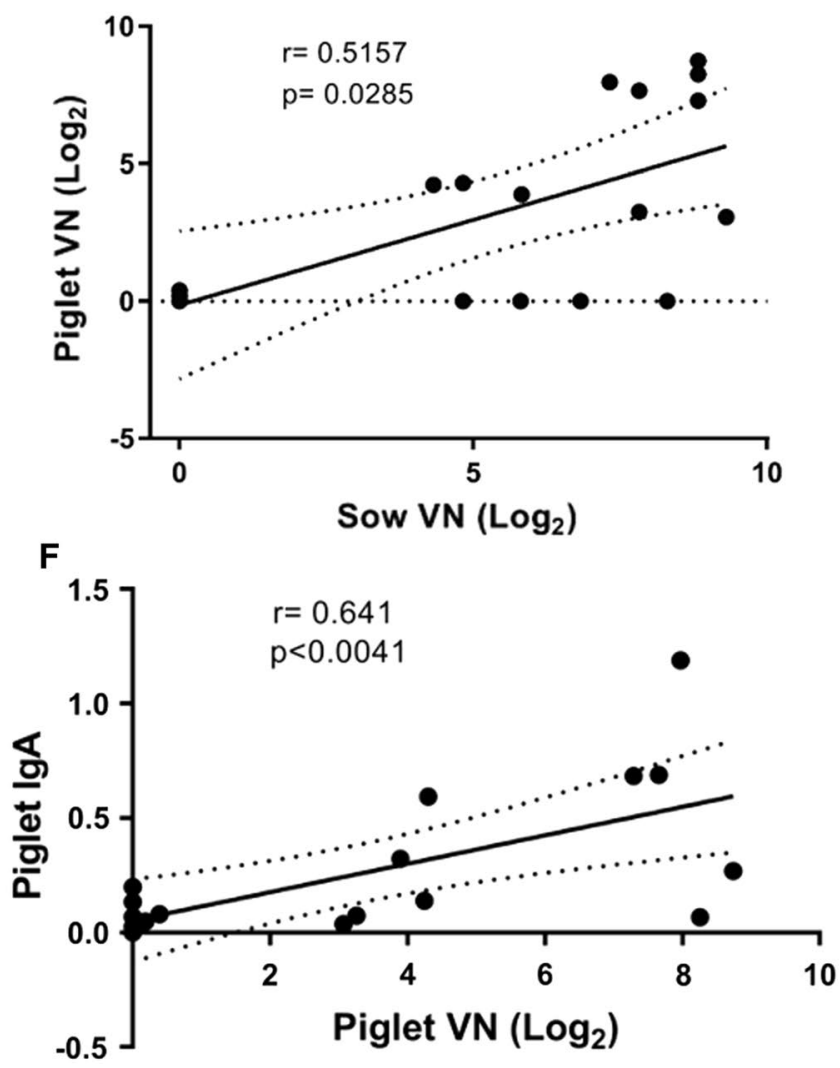

significance level is $<0.01$. S/P, sample-to-positive ratio; the arrowhead represents the day of challenge with PEDV. (D) Correlation of piglet serum NA levels with sow mean NA levels. (E) Correlation of group mean $\mathrm{IgG}$ antibodies in piglet serum with piglet serum NA levels. (F) Correlation of group mean IgA antibodies in piglet serum with piglet serum NA levels. Correlations were calculated using the Spearman method with a 95\% confidence interval using GraphPad Prism 7 

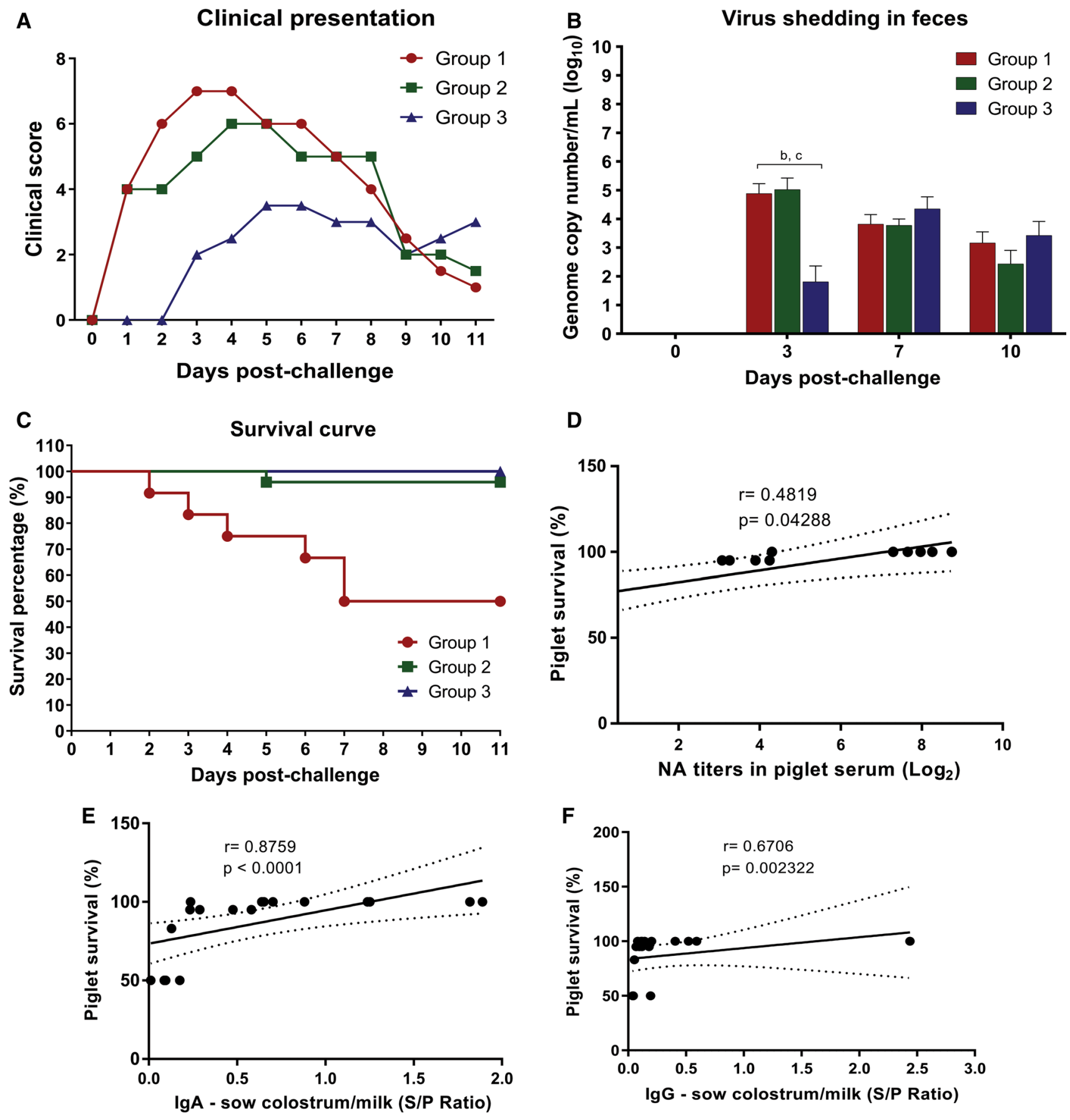

Fig. 4 Clinical presentation, virus shedding, and piglet survival. (A) Daily average group clinical scores recorded after challenge infection with PEDV strain CO13. (B) Virus shedding as determined by PEDV RT-qPCR in piglet feces expressed as $\log _{10}$ genome copy number per milliliter. Data are presented as group means. Error bars represent +/- SEM. Statistical significance was determined using two-way ANOVA, and multiple comparisons were ran using Tukey's test. a, b, and c represent statistical significance for G1 vs. G2, G1 vs. G3, and $\mathrm{G} 2$ vs. G3, respectively. The significance level is $<0.01$. (C) Survival curve demonstrating piglet mortality. The survival curve was gener-

ated using the Kaplan-Meier method. Statistical comparison between groups was performed using the log-rank test in GraphPad Prism 7. The $P$-value was adjusted using the Bonferroni method. (D) Correlation of piglet mortality with piglet group mean NA levels. Correlation of piglet mortality with group mean IgA antibodies in sow colostrum/ milk. (F) Correlation of piglet mortality with group mean IgG antibodies in sow colostrum/milk. Correlations were calculated using the Spearman method with 95\% confidence interval using GraphPad Prism 7 
$(12 / 24 ; 50 \%)$ piglets in control G1 died by day $7 \mathrm{pc}$, while only one piglet $(1 / 24 ; 5 \%)$ in $\mathrm{G} 2$ died on day $5 \mathrm{pc}$, and none of the piglets $(0 / 24 ; 0 \%)$ in $\mathrm{G} 3$ died after the PEDV challenge. Interestingly, when the correlation between antibody levels in piglets and sows was compared to survival rates in piglets, moderate to strong correlations between NA levels in piglet serum $(r=0.4819, P=0.04288)$ and $\operatorname{IgA}$ and $\mathrm{IgG}$ antibody levels in the sow colostrum/milk $(r=0.8759$, $P<0.0001 ; r=0.6706, P=0.002322$, respectively) were observed (Fig. 4D, E and F). Together, these results demonstrate decreased morbidity and mortality in piglets born to immunized gilts and a strong correlation between survival and antibody levels in colostrum and milk.

\section{Discussion}

In the present study, we assessed the ability of ORFV-PEDV$S$ to induce passive immunity against PEDV following immunization of pregnant gilts. One of the goals of the study was to assess whether immunization with ORFV-PEDV-S alone would be sufficient or if live exposure to PEDV would be required for protection of piglets born to immunized gilts. This underlies our experimental design, in which pregnant gilts were either immunized with ORFV-PEDV-S (G2) or with ORFV-PEDV-S followed by oral exposure to live PEDV (G3). Similar to our findings in 3-week-old weaned pigs [11], IM immunization of gilts with ORFV-PEDV-S elicited PEDV-specific IgG, IgA and NAs. As expected, the booster provided by live PEDV exposure in gilts from G3 led to higher antibody responses in serum, colostrum and milk of these animals. Notably, passive transfer of antibodies from gilts to piglets was observed in both $\mathrm{G} 2$ and $\mathrm{G} 3$, as PEDV-specific IgG, IgA and NAs were detected in serum of piglets born to immunized gilts following ingestion of colostrum and milk. NA antibodies detected in piglet serum showed a strong correlation with IgG and IgA levels (Fig. 3E and F). Although the antibody responses elicited by immunization with ORFV-PEDV-S alone were lower in comparison with those induced by immunization with ORFV-PEDV-S followed by live PEDV exposure, the levels of antibodies induced by IM immunization with ORFV-PEDV-S were sufficient to reduce neonatal mortality after oral challenge with PEDV.

Antibodies present in sow colostrum and milk are derived either from serum or produced locally in the mammary tissue [14], and transfer of pathogen-specific antibodies from the sow to the piglet via colostrum and milk is critical for protection against the pathogens during the neonatal phase of the piglet's life. In this context, IgA plays a key role in protection against enteric pathogens such as PEDV, mainly because it is stable to proteolytic degradation in the intestine. By remaining in the gut lumen, pathogen-specific IgA can effectively inhibit/ decrease virus infection/replication in the gut epithelium $[8,15-17]$. The results of this study show that increasing levels of $\operatorname{IgA}$ in milk of immunized animals paralleled lower disease morbidity and mortality in piglets born to immunized gilts after challenge with PEDV. While severe PED and high mortality (50\%) were observed in piglets born to control gilts in G1 (no antibody responses detected), piglets born to gilts in G2 and G3 (high levels of IgA in G2 and even higher in G3) showed less-severe clinical signs and reduced mortality rates $(5$ and $0 \%$, respectively). These results suggest that PEDV-specific IgA detected in piglets in G2 and G3 may have contributed to reducing disease severity and mortality in piglets. The possibility that other antibody isotypes or T cell-mediated immunity may have played a role in protection, however, cannot be formally excluded.

One of the most important observations of our study was the reduced mortality in piglets from G2 (5\% versus 50\% in the control group, G1), which were born to gilts immunized IM with ORFV-PEDV-S. Notably, the lower piglet mortality in this group paralleled S-specific IgG, IgA and NA responses detected in the gilts and passively transferred to piglets. Together, these observations show that parenteral immunization with ORFV-PEDV-S is sufficient to induce protective levels of passive immunity against PEDV. These results corroborate the findings of previous studies in which PEDV specific-antibodies have been detected in serum and colostrum/milk and passively transferred to piglets after parenteral immunization with live, inactivated and/or subunit vaccine candidates [18]. Although the mechanism(s) underlying this phenomenon remain unknown, it is possible that systemic antibodies could be transferred from the sow's serum to colostrum and milk and then to the piglets. Alternatively, the immunomodulatory properties of the ORFV vector used here could potentially lead to migration of antibody-secreting plasma cells to the mammary gland, resulting in local antibody production. Future studies assessing local immune cells in the mammary gland and antibody isotypes in the intestinal lumen of piglets born to immunized sows will be critical for dissecting the precise mechanism(s) underlying the protective immune responses elicited by IM immunization with ORFV-PEDV-S observed here.

In summary, this study demonstrates the ability of ORFVPEDV-S to confer passive immunity against PEDV following immunization of pregnant gilts. Similar to our previous findings in 3-week-old weaned pigs [11], IM immunization of gilts with ORFV-PEDV-S elicited PEDV-specific IgG, IgA and NAs responses. Additionally, passive transfer of antibodies from gilts to piglets was observed, as PEDV-specific IgG, $\operatorname{Ig} \mathrm{A}$ and NAs were detected in serum of piglets born to immunized gilts following ingestion of colostrum and milk. 
Acknowledgements We thank the staff of the SDSU Animal Resource Wing (ARW) for excellent care and handling of animals and for their invaluable help with the animal experiments.

\section{Compliance with ethical standards}

Funding This study was funded by the National Pork Board grant no. 15-034 and in part by the National Institute of Food and Agriculture Hatch project no. SD00517-14.

Conflict of interest The authors declare that there is a patent pending related to this work (US patent pending, P11703US00).

Ethical approval All applicable international, national, and/or institutional guidelines for the care and use of animals were followed. All animal experiments were reviewed and approved by the SDSU IACUC (approval no. 16-003A). This article does not contain any studies with human participants performed by any of the authors.

\section{References}

1. Song D, Park B (2012) Porcine epidemic diarrhoea virus: a comprehensive review of molecular epidemiology, diagnosis, and vaccines. Virus Genes 44(2):167-175. https://doi.org/10.1007/s1126 2-012-0713-1

2. Carvajal A, Argüello H, Martínez-Lobo FJ, Costillas S, Miranda R, GdN PJ, Rubio P (2015) Porcine epidemic diarrhoea: new insights into an old disease. Porcine Health Manag. https://doi. org/10.1186/s40813-015-0007-9

3. Madson DM, Magstadt DR, Arruda PHE, Hoang H, Sun D, Bower LP, Bhandari M, Burrough ER, Gauger PC, Pillatzki AE, Stevenson GW, Wilberts BL, Brodie J, Harmon KM, Wang C, Main RG, Zhang J, Yoon KJ (2014) Pathogenesis of porcine epidemic diarrhea virus isolate (US/Iowa/18984/2013) in 3-week-old weaned pigs. Vet Microbiol 174(1):60-68. https://doi.org/10.1016/j.vetmi c. 2014.09.002

4. Stevenson GW, Hoang H, Schwartz KJ, Burrough ER, Sun D, Madson D, Cooper VL, Pillatzki A, Gauger P, Schmitt BJ (2013) Emergence of Porcine epidemic diarrhea virus in the United States: clinical signs, lesions, and viral genomic sequences. J Vet Diagn Investig 25(5):649-654. https://doi.org/10.1177/10406 38713501675

5. Langel SN, Paim FC, Lager KM, Vlasova AN, Saif LJ (2016) Lactogenic immunity and vaccines for porcine epidemic diarrhea virus (PEDV): historical and current concepts. Virus Res 226:93-107. https://doi.org/10.1016/j.virusres.2016.05.016

6. Leidenberger S, Schröder C, Zani L, Auste A, Pinette M, Ambagala A, Nikolin V, Smit H, Beer M, Blome S (2017) Virulence of current German PEDV strains in suckling pigs and investigation of protective effects of maternally derived antibodies. Sci Rep 7(1):10825. https://doi.org/10.1038/s41598-017-11160-w

7. Bohl EH, Gupta RKP, Olquin MVF, Saif LJ (1972) Antibody responses in serum, colostrum, and milk of swine after infection or vaccination with transmissible gastroenteritis virus. Infect Immun 6(3):289-301

8. Saif LJ, Bohl EH (1979) Passive immunity in transmissible gastroenteritis of swine: immunoglobulin classes of milk antibodies after oral-intranasal inoculation of sows with a live low cell culture-passaged virus. Am J Vet Res 40(1):115-117

9. Song D, Moon H, Kang B (2015) Porcine epidemic diarrhea: a review of current epidemiology and available vaccines. Clin Exp Vaccine Res 4:166-176. https://doi.org/10.7774/cevr.2015.4.2.166

10. Gerdts V, Zakhartchouk A (2016) Vaccines for porcine epidemic diarrhea virus and other swine coronaviruses. Vet Microbiol 206:45-51. https://doi.org/10.1016/j.vetmic.2016.11.029

11. Hain KS, Joshi LR, Okda F (2016) Immunogenicity of a recombinant parapoxvirus expressing the spike protein of Porcine epidemic diarrhea virus. J Gen Virol 97(10):2719-2731. https://doi. org/10.1099/jgv.0.000586

12. Lohse L, Krog JS, Strandbygaard B, Rasmussen TB, Kjaer J, Belsham GJ, Botner A (2016) Experimental infection of young pigs with an early european strain of porcine epidemic diarrhoea virus and a recent US strain. Transbound Emerg Dis 64(5):1380 1386. https://doi.org/10.1111/tbed.12509

13. Okda F, Liu X, Singrey A, Clement T, Nelson J, Christopher-Hennings J, Nelson EA, Lawson S (2015) Development of an indirect ELISA, blocking ELISA, fluorescent microsphere immunoassay and fluorescent focus neutralization assay for serologic evaluation of exposure to North American strains of Porcine Epidemic Diarrhea Virus. BMC Vet Res 11:180. https://doi.org/10.1186/s1291 7-015-0500-Z

14. Bourne FJ, Curtis J (1973) The transfer of immunoglobulins IgG, $\mathrm{IgA}$ and IgM from serum to colostrum and milk in the sow. Immunology 24(1):157-162

15. Arriba DML, Carvajal A, Pozo J, Rubio P (2002) Mucosal and systemic isotype-specific antibody responses and protection in conventional pigs exposed to virulent or attenuated porcine epidemic diarrhoea virus. Vet Immunol Immunopathol 85:85-97

16. Poonsuk K, Giménez-Lirola LG, Zhang J, Arruda P (2016) Does circulating antibody play a role in the protection of piglets against porcine epidemic diarrhea virus? PLoS One 11:1-17. https://doi. org/10.1371/journal.pone.0153041

17. Chattha KS, Roth JA, Saif LJ (2015) Strategies for design and application of enteric viral vaccines. Annu Rev Anim Biosci 3(1):375-395. https://doi.org/10.1146/annurev-animal-02211 4-111038

18. Paudel S, Park JE, Jang H, Hyun BH, Yang DG (2014) Evaluation of antibody response of killed and live vaccines against porcine epidemic diarrhea virus in a field study. Vet Q 34(4):194-200. https://doi.org/10.1080/01652176.2014.973999 